Nanoscale Aovances

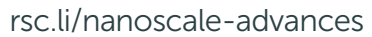
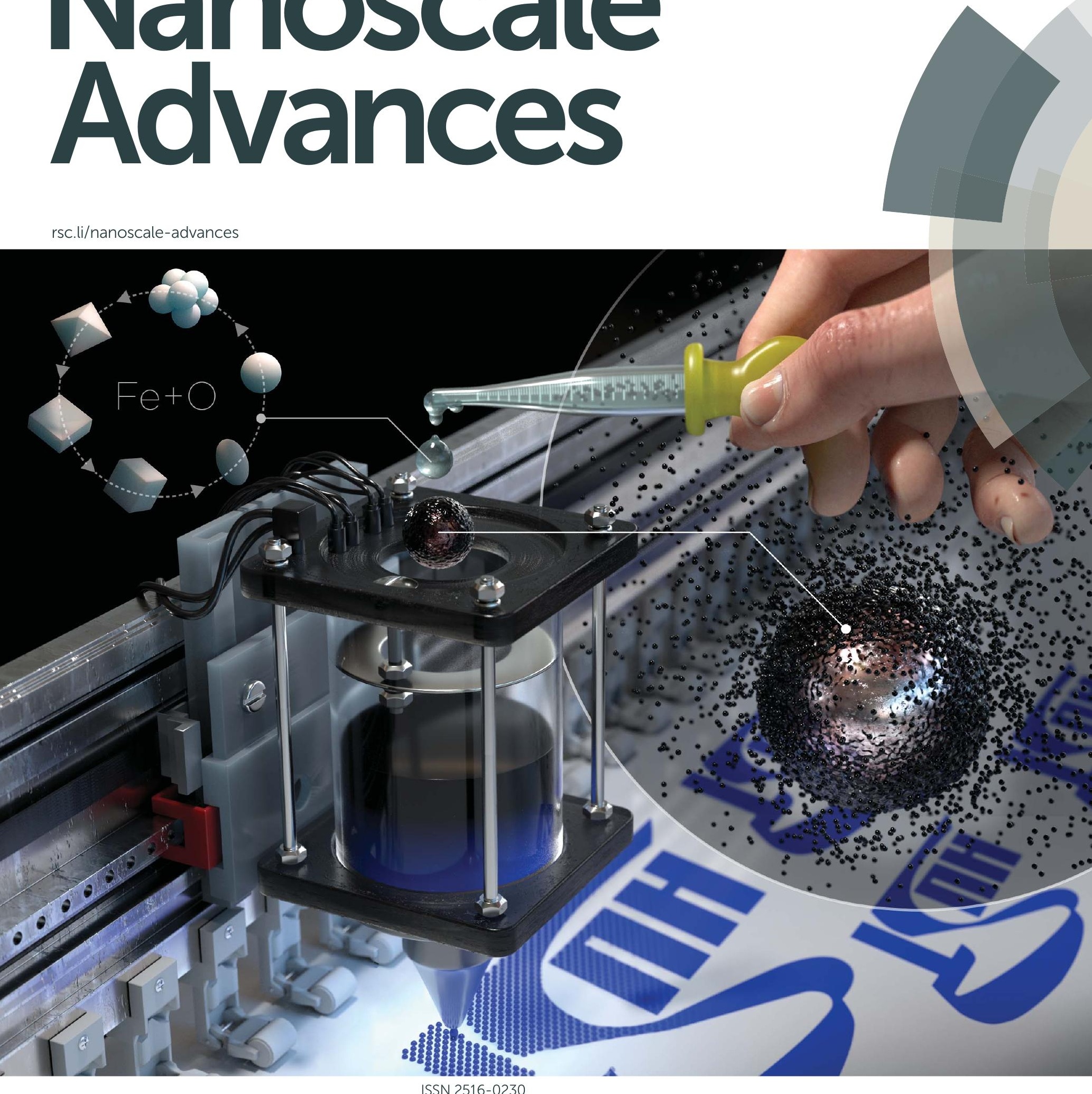
Check for updates

Cite this: Nanoscale Adv., 2019, 1, 2523

\title{
Shape transformation and self-alignment of Fe-based nanoparticles $\uparrow$
}

\author{
Jeongmin Hong, (D) *ab Qiang Luo, ${ }^{a}$ Daesung Jung, ${ }^{c}$ Soong-Geun Je, ${ }^{d}$ Yooseok Kim, ${ }^{e}$ \\ Mi-Young Im, ${ }^{d}$ Chan-Cuk Hwang, ${ }^{c}$ Sakhrat Khizroev, ${ }^{f}$ Seungjun Chung ${ }^{g}$ \\ and Long You (iD a
}

New types of functional material structures will emerge if the shape and properties are controlled in threedimensional nanodevices. Possible applications of these would be nanoelectronics and medical systems. Magnetic nanoparticles (MNPs) are especially important in electronics such as magnetic storage, sensors, and spintronics. Also, in those that are used as magnetic resonance imaging contrasts, and tissue specific therapeutic agents, as well as in the labeling and sorting of cells, drug delivery, separation of biochemical products, and in other medical applications. Most of these applications require MNPs to be chemically stable, uniform in size, and controllable in terms of their magnetic properties and shape. In this paper three new functions of iron (Fe)-based nanoparticles are reported: shape transformation, oxidation prevention, and self-alignment. The shape of the Fe nanoparticles could be controlled by changing their oxidation states and properties by using a nanocarbon coating. Full field $\mathrm{X}$-ray microscopy using synchrotron radiation revealed controllable magnetic properties of MNPs at the $L_{3}$ edge which depended on the oxidation states. Then, inkjet printing was successfully performed to deposit a uniform layer of MNPs by the size.

Received 7th March 2019

Accepted 12th May 2019

DOI: 10.1039/c9na00146h

rsc.li/nanoscale-advances reduced down to the sub-10 $\mathrm{nm}$ range. For example, one of the important applications of superparamagnetic NPs is their use as drug-delivery agents to overcome the blood-brain barrier under controllable magnetic fields. ${ }^{18,19}$ However, MNPs tend to aggregate into large clusters, and thus, lose specific properties associated with single domain magnetic nanostructures. ${ }^{20}$ Therefore, a new, efficient approach to achieve adequately controllable magnetic properties of MNPs needs to be found.

Iron (Fe) shows superior magnetic properties which are important for spintronics and medical applications. However, it is easily oxidized, which changes its physical properties, by using phase transitions. Pure Fe NPs covered by nanocarbon shell structures such as carbon nanotubes (CNTs) and graphene could not only protect the oxidation, but manipulate the carbon's own properties. ${ }^{21}$ The production of pure Fe NPs is challenging, but after systematic synthesis of a nanocarbon shell, the properties of Fe can present without further oxidation. $^{22,23}$ The use of the superior properties of nanocarbon structures and interfaces could be useful for many applications because of the magneto-electric effects. ${ }^{24-26}$

Although the use of NPs in solution can be controlled well, MNPs are very challenging to align in a dry form because of its interactions between each particle. ${ }^{24}$ Drop casting and spray methods are very commonly used to deposit MNPs onto substrates. Using inkjet printing technology it is possible to align MNPs in multiple dimensions. Printing of MNPs makes it possible to align them in two-dimensional and threeSeoul 02792, South Korea

$\dagger$ Electronic supplementary information (ESI) available. See DOI: 10.1039/c9na00146h 
dimensional (3D) structures if it is deposited in a controllable way. ${ }^{27}$ Using the optimal conditions for inkjet printing, a drop of the liquid's suspended particulate matter is deposited to form a ring on the substrate. These effects could control the alignment of a same size distribution of the NPs because of the fluid dynamics.

In this paper, a unique way to modulate MNPs by controlling the size, shape, and magnetic properties is presented. To prevent oxidation, a nanocarbon shell was used to cover Fe structures. For oxides and pure Fe states, the magnetic properties of the nanocarbon have not been well studied using characterizing magnetometry. ${ }^{28,29}$ Magnetic microscopy such as magnetic force microscopy limits phase imaging because of its topographical effects on the MNPs as described in the ESI. $\dagger$ Using synchrotron radiation, full field X-ray microscopy was successfully performed to present element specific magnetic imaging using X-ray magnetic circular dichroism (XMCD). The Fe-based particle imaging could distinguish pure $\mathrm{Fe}$ and $\mathrm{Fe}-\mathrm{O}$ at the $\mathrm{L}_{3}$ edge. Then, the particles were printed onto rigid, and even flexible, substrates using inkjet printing technology.

\section{Results and discussion}

\section{Fe-O synthesis}

The synthesis started with magnetic NPs obtained using a conventional method. ${ }^{29}$ The size of the magnetic NPs varied from $5 \mathrm{~nm}$ to $200 \mathrm{~nm}$ as shown in Fig. 1. From the results of the coagulation experiment, it was found that the particles coagulated very easily by applying a magnetic field. The coagulation of MNPs was a more stable if the coagulation stabilized. When the fabrication conditions such as $\mathrm{pH}$ and process temperature were changed, the particles transformed to other stable forms of iron oxide, such as maghemite $\left(\gamma-\mathrm{Fe}_{2} \mathrm{O}_{3}\right)$ and hematite $(\alpha$ $\mathrm{Fe}_{2} \mathrm{O}_{3}$ ). Five types of shape transformation of MNPs were observed: pyramidal, square, hybrid (coexisting with circular and square structures), and oval structures as reported previously in the literature. ${ }^{30}$

A controllable synthesis of nanocrystalline iron oxide, particularly, of the pyramidal shape of MNPs was performed. Fig. 1a-f show the transformation cycle. The initial magnetite was oxidized to the transition state before pyramid formation, and was then transformed into the square structure. After further oxidized, this structure saturates to the mixed states of the circular, square, and oval structures. It should be noted that the different oxidation levels during synthesis resulted in the coarsening of the precipitated NPs.

Bi-pyramidal structures were the most common structures as reported. ${ }^{31-34}$ However, a single pyramid is also very important, especially for magnetic sensors to focus the magnetic field along the direction of the apex. Ideally, octahedral and tetrahedral structures, indicative of the formation of self-assembled pyramidal nanodevices, can be seen in the X-ray diffraction (XRD) patterns shown in the ESI. $\dagger$ A unique combination of octahedral and tetrahedral lattice structures was found, resulting in perfectly shaped pyramidal pits of iron oxide MNPs.

\section{Vibrating sample magnetometry}

Vibrating sample magnetometry (VSM) shows superparamagnetic behaviour in relation to the levels of oxidation, as shown in Fig. 1c. The unique structure could be used for an

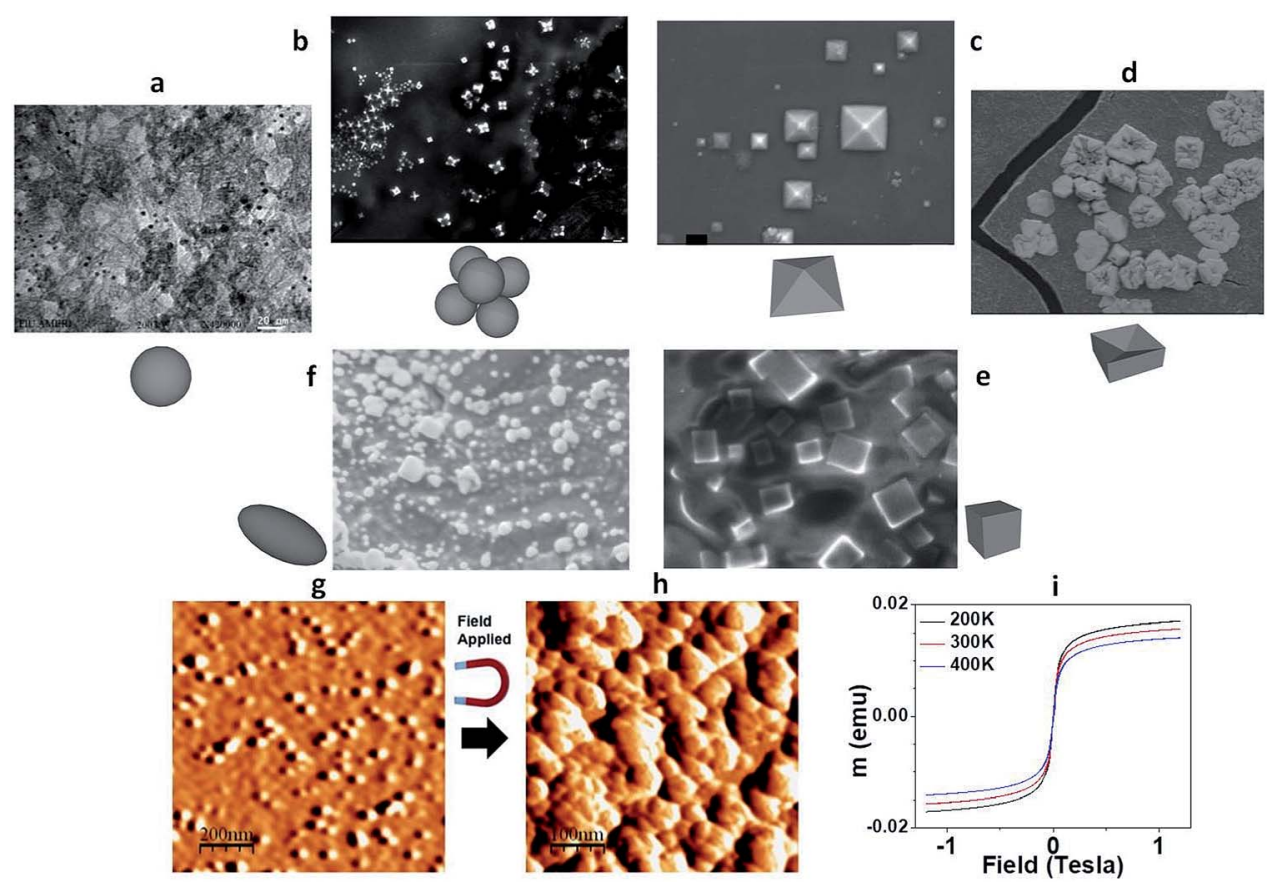

Fig. 1 Formation and magnetic properties of MNPs. The shape transformation cycle from circular to hybrid structures of MNPs: (a) circular shapes, (b) star shapes, (c) pyramidal shapes, (d) negative pyramidal shapes, (e) square shapes, and (f) mixed states. Magnetic properties of MNPs: AFM images of MNPs (g) before and (h) after the magnetic field was applied, (i) $m-H$ loops of MNPs using different temperature ranges. 
intense magnetic field generation and sensing due to its unique structure. The octadecahedral $\alpha-\mathrm{Fe}_{2} \mathrm{O}_{3}$ nanocrystals with a hexagonal pyramid shape were synthesised by packing truncated nanodots into several different superstructures. The findings are of fundamental importance to understanding the morphology and growth of $\alpha-\mathrm{Fe}_{2} \mathrm{O}_{3}$ nanostructures. Improved control of the shape evolution of hematite particles will be studied to examine the direct correlation between exposed facets and facet controlled properties in a future study.

\section{Fe-C synthesis}

For the self-aligned NPs using inkjet printing, the NPs need to be chemically stable. To avoid the oxidation state, nanocarbon coating was applied by precisely controlling the carbon states such as graphene, nanotubes, and others. The $\mathrm{Fe}-\mathrm{C}$ composites were fabricated by applying a high energy milling of iron at a low temperature. The method was able to prevent oxidation and may promote Fe NPs without any oxide or impurity. It was noted that cryo-milling in an inert atmosphere had the ability to produce free NPs of metals with minimum contamination. ${ }^{32}$ The present experiments were conducted using Fe powders of 99.999\% purity in a specially designed mill under pure argon. ${ }^{32}$ The curing and milling was possible at a temperature of $\sim 150 \mathrm{~K}$ with the use of liquid nitrogen $\left(\mathrm{LN}_{2}\right)$ cooling. ${ }^{32}$ The average particle size was reduced substantially, because of the cryomilling, from the approximate $20 \mu \mathrm{m}$ size of the as-received powder to a size of $\sim 10 \mathrm{~nm}$ after $3 \mathrm{~h}$ of milling. Then, carbon coating was performed using chemical vapour deposition. ${ }^{21}$ Scanning electron microscopy images in Fig. 2a-d show the morphology of $\mathrm{Fe}-\mathrm{C}$ structures with the changes of the growth temperature from $1000 \mathrm{~K}, 1100 \mathrm{~K}, 1200 \mathrm{~K}$, to $1250 \mathrm{~K}$, respectively. One of the particles was chosen for thin film energy dispersive X-ray spectroscopy analysis with a fine probe which again indicated that oxygen was absent in the NPs. Furthermore, the electron energy loss spectroscopy (EELS) did not show the presence of oxide (see the ESI $\uparrow$ for further details). However, the analysis of EELS spectra from the edges indicates $\sim 0.1 \%$ of oxygen solubility in Fe.

The results of the Raman analyses showed two easily distinguished peaks which were the D- and G-peaks. From the growth temperature at $1000 \mathrm{~K}$, the main peak of the Raman spectra showed both D- and G-peaks. As shown in Fig. 2e, about $60 \%$ of particles with a diameter of several hundreds of nanometers showed the presence of CNT peaks, and also mixed states of CNT and graphene at $1100 \mathrm{~K}$. The graphene state was also well defined at $1200 \mathrm{~K}$, but a small CNT peak was also observed. At $1250 \mathrm{~K}$, an excellent graphene peak was observed and the roughness was good with tiny CNT peaks as shown in Fig. 2e.

To distinguish whether the graphene layers protected the $\mathrm{Fe}$ particles from oxidation, images of the MNPs were obtained using XMCD because it allowed element specific measurement. For this purpose, full-field magnetic transmission soft X-ray microscopy was used. ${ }^{35}$ Fig. $2 \mathrm{f}$ shows the XMCD image of the Fe-C core-shell measured at the $\mathrm{Fe}-\mathrm{L}_{3}$ absorption edge (708 eV). Fig. $2 \mathrm{f}$ is a differential image of two images taken at perpendicular magnetic fields of \pm 2700 Oe, and the dark contrast indicated that the MNPs exhibited magnetic properties of Fe, suggesting that the monolayer graphene membrane served as a coating to prevent any strong reaction as was expected.

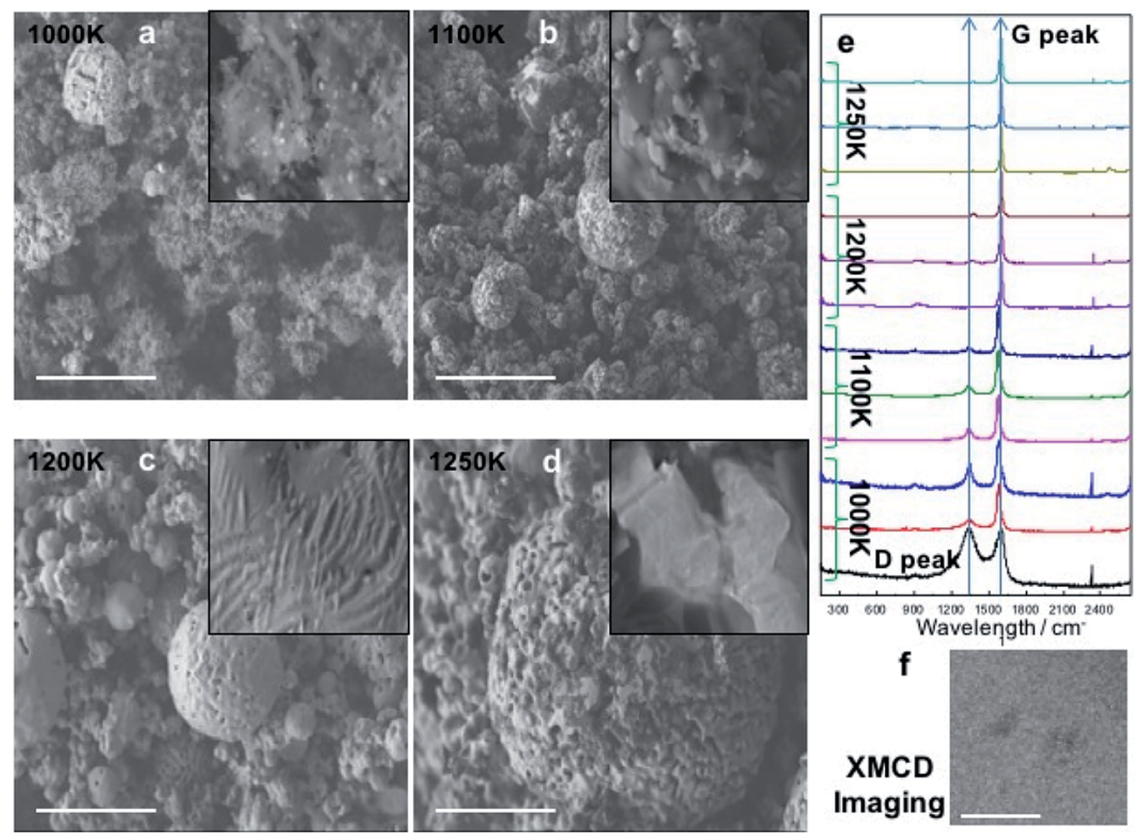

Fig. 2 Fe-C core-shell structures. SEM images of Fe-C core-shell structures grown at $1000 \mathrm{~K}$ (a), $1100 \mathrm{~K}$ (b), $1200 \mathrm{~K}$ (c), and $1250 \mathrm{~K}$ (d) (scale bars are $1 \mu \mathrm{m}$ ). (e) Raman peaks at different synthesis temperatures and times. Notice the change of D- and G-peaks by varying the temperature ranges from $1000 \mathrm{~K}$ to $1250 \mathrm{~K}$, respectively. (f) A full field magnetic micrograph of the Fe-C core-shell using synchrotron radiation. The dark regions represent the XMCD of the Fe-C core-shells (scale bar is $500 \mathrm{~nm}$ ). 


\section{Ink-jet printing process}

Although drop casting is a well-known method to deposit MNPs onto dry substrates, it is hard to control the distribution of MNPs depending on their size because of the large volume that drops over a few $\mu \mathrm{L}$. However, inkjet printing allowed a more precise patterning, with less volume of droplets $(\sim \mathrm{pL})$, from a nozzle with a diameter of tens of $\mu \mathrm{m}$. This strategy had several advantages such as low-cost, easy access to electronic devices, and a precise material dispersion technique with non-contact deposition on a variety of substrates. ${ }^{27}$ After determining the optimal conditions, the prepared MNPs were inkjet printed onto rigid (silicon, Si) and flexible (poly(dimethylsiloxane), PDMS) substrates.

The experimental details are described in the Materials and methods section. Fig. 3a shows a schematic and an optically captured image of Fe-C MNPs jetting. By optimizing the ejection conditions including voltage pulse amplitude and duration, the droplets in-flight from single nozzle were well defined spheres. Fig. $3 \mathrm{~b}$ shows that the particles were aligned by size because of the coffee ring effect after dropping onto the substrates. The results with the deposition of $\mathrm{Fe}-\mathrm{C}$ particles showed that the structures were well aligned. The size controllable effects are discussed more in detail in the ESI. $\uparrow$ Using inkjet printing, the size and distribution of MNPs could be controlled as shown in Fig. 3c. Fig. 3d shows that the MNPs were well aligned in a uniform way. The size distribution showed that the uniform arrays of NPs having a diameter of sub $10 \mathrm{~nm}$ were aligned in the middle region. When they were deposited on a PDMS substrate, it was observed that the MNPs were well aligned. The left and right images of Fig. 3d show scanning electron microscopy (SEM) and atomic force microscopy (AFM) images of MNPs deposited on PDMS substrate, respectively.

\section{Materials and methods}

\section{Nanomagnetic particle fabrication}

Nanomagnetic particles were fabricated using coprecipitation from a solution of ferrous and ferric mixed salts in the ratio of $1: 2$ under the ambient conditions of the bulk aqueous solution and surfactant systems. ${ }^{\mathbf{1 1 , 1 6 , 2 4}}$ De-ionized water $(300 \mathrm{~mL})$ was deaerated in a three-necked flask by stirring with the application of ultra-high purity nitrogen. First, $2 \mathrm{~g}$ of hydrated ferrous chloride $\left(\mathrm{FeCl}_{2} \cdot 2 \mathrm{H}_{2} \mathrm{O}\right.$ ), was mixed with $5.4 \mathrm{~g}$ of hydrated ferric chloride $\left(\mathrm{FeCl}_{3} \cdot 6 \mathrm{H}_{2} \mathrm{O}\right)$, in $300 \mathrm{~mL}$ of water, and then dissolved at $30-40{ }^{\circ} \mathrm{C}$. Then, a solution of $5 \mathrm{~g}$ sodium hydroxide in water at $50{ }^{\circ} \mathrm{C}$ was added at a constant stirring rate of $2000 \mathrm{rpm}$, and a black magnetite precipitate was deposited.
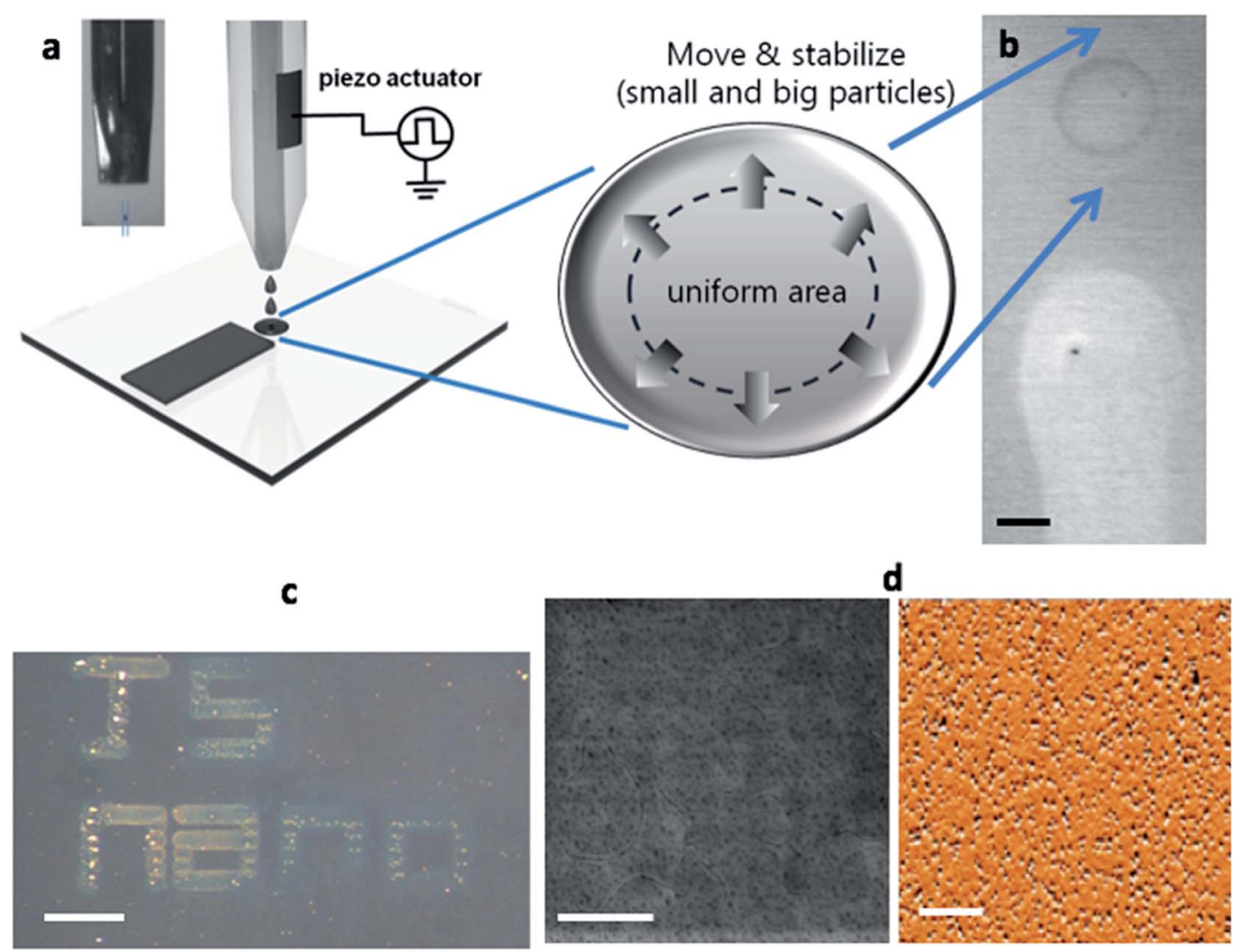

Fig. 3 Distribution of the MNPs using inkjet printing. (a) A schematic of the inkjet jetting image which shows micro-sized Fe-C solution drops. The volume of an ejected single droplet was $60 \mathrm{pL}$ from a nozzle with a diameter of $30 \mu \mathrm{m}$. (b) An optical microscopy image of an MNP single drop (top) and line (bottom) formation onto a Si substrate. When exploiting the coffee ring effect, the particles were aligned by a uniform size. (c) Using optimal drops and lines, drop-on demand printing was performed (Scale bar is $2 \mathrm{~mm}$ ). (d) The resulting images of uniformly arrayed Fe-C structures onto PDMS using SEM (L) and AFM (R) (scale bar is $2 \mu \mathrm{m}$ ). 


\section{Transmission electron microscopy}

A transmission electron microscope (TEM; Philips CM200) with an acceleration voltage of $200 \mathrm{kV}$ was used to take the TEM images. A field-emission gun equipped with an imaging filter (Gatan, GIF200) running DigitalMicrograph ${ }^{\mathrm{TM}}$ (Gatan) software was used. The TEM samples were prepared by placing a drop of mini-emulsion onto a carbon-coated copper $(\mathrm{Cu})$ grid under ambient conditions.

\section{X-ray diffraction}

The XRD measurements were made using a powder X-ray diffractometer (Siemens, D-5000) equipped with a $\mathrm{Cu}$ anode operated at $40 \mathrm{kV}$ and $40 \mathrm{~mA}$. The XRD patterns were collected using a step size of $0.01^{\circ}$ and a scan rate of 1 step per s. Data were analyzed from the Powder Diffraction File (PDF).

\section{Scanning electron microscopy}

The SEM (Jeol 9000F) was performed at $15 \mathrm{kV}$ and a working distance of $5 \mathrm{~mm}$.

\section{Vibrating sample magnetometry}

The $m-H$ loop measurements were performed using the VSM option of a cryogenic physical property measurement system (PPMS, Quantum Design) with a 9 T superconducting magnet. Samples were mounted in a regular tube holder. To optimize the touchdown process, the samples were mounted with an upward offset of $35 \mathrm{~mm}$. The magnetic field was swept at the rate of $15 \mathrm{Oe} \mathrm{s}^{-1}$.

\section{Magnetic force microscopy}

The AFM (Veeco, MultiMode system) study was performed in non-contact mode. High sensitivity magnetic tunnel junction probes $\left(2 \mathrm{nT} \mathrm{Hz}^{-1}\right)$ at $50 \mathrm{~Hz}$ were provided by JS Nanotechnologies (San Jose, CA). The particle imaging was performed in a standard scanning probe microscopy system.

\section{XM-1 microscopy}

The XMCD was carried out using full-field soft X-ray transmission microscopy at the Lawrence Berkeley National Laboratory, Advanced Light Source, Beamline 6.1.2 along the Fe (708 eV) absorption edge. For the transmission measurement, the MNPs were prepared on a transparent silicon nitride membrane.

\section{Inkjet printing}

The MNPs were printed on a $60{ }^{\circ} \mathrm{C}$ substrate using a customized drop-on-demand piezoelectric-type inkjet printing system with MJ-AT-01 nozzles (MicroFab Corp). The experimental conditions were based on the following parameters: concentration, type of solvent, substrate temperature, and drop spacing. Different conditions were tried by changing the concentrations $\left(0.1,0.5\right.$, or $\left.1.0 \mathrm{~g} \mathrm{~mL}^{-1} \mathrm{~s}^{-1}\right)$, of different solvents such as: isopropyl alcohol or acetone. The temperature range of the substrates was 50,60 , and $70^{\circ} \mathrm{C}$. The variations of drop spacing were performed from 30 to $180 \mu \mathrm{m}$. Using the optimal results, MNPs were deposited onto different substrates such as rigid and flexible ones.

\section{Conclusions}

Three important milestones were found for MNPs. Firstly, the formation of self-assembled 3D pyramidal NPs could be optimized by changing the process conditions. The formation of four phases of the self-assembled 3D nanodevices such as pyramidal, square, hybrid, and circular nanostructures could be controlled. Secondly, Fe-C particles could be used to prevent oxidation and showed strong XMCD images at the $\mathrm{Fe} \mathrm{L}_{3}$ edge. That means that carbon prevents the penetration of any atoms into the Fe core. Lastly, inkjet printing was applied to align the MNPs. The MNPs were aligned in a size controllable and uniform way onto flexible substrates. These results pave the way for their use in the field of NP-based future electronics and smart medicine.

\section{Conflicts of interest}

There are no conflicts to declare.

\section{Acknowledgements}

This work is supported by the National Natural Science Foundation of China under Award number 61674062. The work was supported by the US Department of Energy, Office of Basic Energy Sciences, Division of Materials Sciences and Engineering under Contract No. DE-AC02-05CH11231. The Authors would like to thank JS Nanotechnologies LLC (http:// www.jsnanotech.com) for their support (Contract No. JS-Nano1901110). This work was also supported by the National Research Foundation of Korea supported by the Korean Government (Ministry of Science and ICT) \#2018R1A5A6075964, \#2017R1A2B2003928, and \#2017M3A7B4049173. The Authors acknowledge the financial support from Air Force Office of Scientific Research (AFOSR, USA) under Award number FA9550-18-1-0527 and the National Science Foundation (NSF) under Award numbers 0939514 and 1810270.

\section{Notes and references}

1 G. M. Whitesides, et al.,Molecular Self-Assembly and Nanochemistry: a Chemical Strategy for the Synthesis of Nanostructures, Science, 1991, 254(5036), 1312-1319.

2 Y. Sun and Y. Xia, Shape-controlled synthesis of gold and silver nanoparticles, Science, 2002, 298(5601), 2176-2179.

3 K. L. Prime and G. M. Whitesides, Self-assembled organic monolayers: model systems for studying adsorption of proteins at surfaces, Science, 1991, 252(5009), 1164-1167.

4 C. Granqvist, R. Buhrman, J. Wyns and A. Sievers, FarInfrared Absorption in Ultrafine Al Particles, Phys. Rev. Lett., 1976, 37(10), 625-629. 
5 P. Yu, Y. Yao, J. Wu, X. Niu, A. L. Rogach and Z. Wang, Effects of Plasmonic Metal Core-Dielectric Shell Nanoparticles on the Broadband Light Absorption Enhancement in Thin Film Solar Cells, Sci. Rep., 2017, 7(1), 7696.

6 C. A. S. Batista, R. G. Larson and N. A. Kotov, Nonadditivity of nanoparticle interactions, Science, 2015, 350(6257), 1242477.

7 M. A. Mitchnick, D. Fairhurst and S. R. Pinnell, Microfine zinc oxide (Z-cote) as a photostable UVA/UVB sunblock agent, J. Am. Acad. Dermatol., 1999, 40(1), 85-90.

8 D. Llamosa, M. Ruano, L. Martinez, A. Mayoral, E. Roman, M. García-Hernández and Y. Huttel, The ultimate step towards a tailored engineering of core@shell and core@shell@shell nanoparticles, Nanoscale, 2014, 6, 13483-13486.

9 M. E. Akerman, W. C. Chan, P. Laakkonen, S. N. Bhatia and E. Ruoslahti, Nanocrystal targeting in vivo, Proc. Natl. Acad. Sci. U. S. A., 2002, 99(20), 12617-12621.

10 A. Fu, C. M. Micheel, J. Cha, H. Chang, H. Yang and A. P. Alivisatos, Discrete nanostructures of quantum dots/ Au with DNA, J. Am. Chem. Soc., 2004, 126(35), 10832-10833.

11 A.-H. Lu, E. L. Salabas and F. Schüth, Magnetic Nanoparticles: Synthesis, Protection, Functionalization, and Application, Angew. Chem., Int. Ed., 2007, 46(8), 1222-1244.

12 G. Reiss and A. Hutten, Magnetic Nanoparticles, in Handbook of Nanophysics: Nanoparticles and Quantum Dots, ed. D. K. Sattler, CRC Press, 2010, pp. 11-1-19-1.

13 B. Gleich and J. Weizenecker, Tomographic imaging using the nonlinear response of magnetic particles, Nature, 2005, 435(7046), 1214-1217.

14 K. E. Scarberry, E. B. Dickerson, J. F. McDonald and Z. J. Zhang, Magnetic Nanoparticle-Peptide Conjugates for in Vitro and in Vivo Targeting and Extraction of Cancer Cells", J. Am. Chem. Soc., 2008, 130(31), 10258-10262.

15 X. Meng, H. C. Seton, L. T. Lu, I. A. Prior, N. T. K. Thanh and B. Song, Magnetic CoPt nanoparticles as MRI contrast agent for transplanted neural stem cells detection, Nanoscale, 2011, 3(3), 977-984.

$16 \mathrm{~S}$. Kralj and D. Makovec, Magnetic Assembly of Superparamagnetic Iron Oxide Nanoparticle Clusters into Nanochains and Nanobundles, ACS Nano, 2015, 9(10), 9700-9707.

17 J.-P. Wang and J. Bai, High-magnetic-moment core-shelltype FeCo-Au/AgFeCo-Au/Ag nanoparticles, Appl. Phys. Lett., 2005, 87, 152502.

18 R. Qiao, Q. Jia, S. Hüwel, R. Xia, T. Liu, F. Gao, H.-J. Galla and M. Gao, Receptor-Mediated Delivery of Magnetic Nanoparticles across the Blood-Brain Barrier, ACS Nano, 2012, 6(4), 3304-3310.

19 M. A. Busquets, A. Espargaró, R. Sabaté and J. Estelrich, Magnetic Nanoparticles Cross the Blood-Brain Barrier: When Physics Rises to a Challenge, Nanomaterials, 2015, 5(4), 2231-2248.

20 M. Zhang, F. Xiao, X. Z. Xu and D. S. Wang, Novel ferromagnetic nanoparticle composited PACIs and their coagulation characteristics, Water Res., 2011, 46(1), 127-135.

21 J. Hong, H. N. Hwang, A. T. N'Diaye, J. Liang, G. Chen, Y. Park, L. T. Singh, Y. G. Jung, J. H. Yang, J. I. Jeong,
A. K. Schmid, E. Arenholz, H. Yang, J. Bokor, C. C. Hwang and L. You, The interfacial spin modulation of graphene on $\mathrm{Fe}(111), 2018$, ArXiv 1811.09773.

22 F. Yu and V. C. Yang, Size-tunable synthesis of stable superparamagnetic iron oxide nanoparticles for potential biomedical applications, J. Biomed. Mater. Res. A, 2011, 92(4), 1468-1475.

23 Z. Kelgenbaeva, E. Omurzak, S. Takebe, S. Sulaimankulova, Z. Abdullaeva, C. Iwamoto and T. Mashimo, Synthesis of pure iron nanoparticles at liquid-liquid interface using pulsed plasma, J. Nanoparticle Res., 2014, 16, 2603.

24 H. K. D. Kim, L. T. Schelhas, S. Keller, J. L. Hockel, S. H. Tolbert and G. P. Carman, Magnetoelectric control of superparamagnetism, Nano Lett., 2013, 13, 884-888.

25 R. Guduru, P. Liang, C. Runowicz, M. Nair, V. Atluri and S. Khizroev, Magneto-electric Nanoparticles to enable fieldcontrolled high-specificity drug delivery to eradicate ovarian cancer cells, Sci. Rep., 2013, 3, 2953.

26 M. Nair, R. Guduru, P. Liang, J. Hong, V. Sagar and S. Khizroev, Externally controlled on-demand release of anti-HIV drug using magneto-electric nanoparticles as carriers, Nat. Commun., 2013, 4, 1707.

27 S. Chung, J. Lee, H. Song, S. Kim, J. Jeong and Y. Hong, Inkjet-printed stretchable silver electrode on wave structured eleastomeric substrate, Appl. Phys. Lett., 2011, 98(15), 153110.

28 L. T. Kuhn, A. K. Geim, J. G. S. Lok, P. Hedegard, K. Ylanen, J. B. Jensen, E. Johnson and P. E. Lindelof, Magnetisation of isolated single crystalline Fe-nanoparticles measured by a ballistic Hall micro-magnetometer, Eur. Phys. J. D, 2000, 10, 259-263.

29 A. K. Gupta and M. Gupta, Synthesis and surface engineering of iron oxide nanoparticles for biomedical applications, Biomaterials, 2005, 26(18), 3995-4021.

30 A. Lassoued, B. Dkhil, A. Gadri and S. Ammar, Control of the shape and size of iron oxide (alpha- $\mathrm{Fe}_{2} \mathrm{O}_{3}$ ) nanoparticles synthesized through the chemical precipitation method, Results Phys., 2017, 7, 3007-3015.

31 C. S. Tiwary, S. Kashyap, K. Biswas and K. Chattopadhyay, Synthesis of pure iron magnetic nanoparticles in large quantity, J. Phys. D: Appl. Phys., 2013, 46(38), 385001.

32 S. Hosseinzadeh, A. Yazdani and R. Khordad, Pure iron nanoparticles prepared by electric arc discharge method in ethylene glycol, Eur. Phys. J. Appl. Phys., 2012, 59(3), 30401.

33 M. Lin, L. Tng, T. Lim, M. Choo, J. Zhang, H. R. Tan and S. Bai, Hydrothermal synthesis of octadecahedral Hematitie (alpha- $\mathrm{Fe}_{2} \mathrm{O}_{3}$ ) nanoparticles: an expitaxial growth from goethite (alpha-FeOOH), J. Phys. Chem. C, 2014, 118, 10903-10910.

34 X.-F. Qu, G.-T. Zhou, Q.-Z. Yao and S.-Q. Fu, Aspartic-acidassisted hydrothermal growth and properties of magnetite octahedrons, J. Phys. Chem. C, 2010, 114, 284-289.

35 S.-G. Je, M.-S. Jung, M.-Y. Im and J.-I. Hong, Electric current control of creation and annihilation of sub-100-nm magnetic bubbles examined by full-field transmission soft X-ray microscopy, Curr. Appl. Phys., 2018, 18(11), 1201-1204. 